\title{
An Instability-driven Dynamo for Gamma-ray Burst Black Holes
}

\author{
Rafael Ángel Araya-Góchez ${ }^{1}$ \\ Laboratorio de Investigaciones Astrofísicas, Escuela de Física \\ Universidad de Costa Rica, San José, Costa Rica.
}

\begin{abstract}
We show that an MHD-instability driven dynamo (IDD) operating in a hot accretion disk is capable of generating energetically adequate magnetic flux deposition rates above and below a mildly advective accretion disk structure. The dynamo is driven by the magnetorotational instability (MRI) of a toroidal field in a shear flow and is limited by the buoyancy of 'horizontal' flux and by reconnection in the turbulent medium. The efficiency of magnetic energy deposition is estimated to be comparable to the neutrino losses although an MHD collimation mechanism may deem this process a more viable alternative to neutrino-burst-driven models of $\gamma$-ray bursts.
\end{abstract}

\section{INTRODUCTION}

The combined redshift and fluence measurements of at least five $\gamma$-ray burst sources plus very large photon energy detections in certain bursts and tight size constraints derived from the rapid risetimes of burst triggers strongly suggest that the release of energy is highly focused by the central engine that propels a $\gamma$ -

ray burst. In spite of the very large $\gamma$-ray energy requirements, the efficiency of energy deposition into electromagnetic channels is likely to be very poor if the burst is driven by a neutrino burst in analogy to the processes thought to give birth to supernovæ (MacFadyen \& Woosley 1998, 1999) or if the burst involves major energy losses to gravitational radiation such as might be the case in compact object merger scenarios (Rasio and Shapiro 1994, Davies et al. 1994, Ruffert \& Janka 1998). Indeed, these measurements pose a serious energy budget problem for arguably all gravitational collapse powered models of $\gamma$-ray bursts if the energy release is not moderately collimated.

An attractive solution to this problem starts with a (directional) Poynting-flux dominated outflow (Thompson 1994, Mészáros \& Rees 1997) under the premise that such a flow may carry very little baryonic contamination if deposited along a centrifugally (or gravitationally) evacuated funnel such as the angular momentum

1) e-mail: araya@twinkie.gsfc.nasa.gov 
axis of a black hole-accretion disk system. Yet, formal motivation for an external field of the desired strength and topology has yet to be investigated in this setting.

We motivate a reasonable set of heuristic two dimensional dynamo equations for magnetic field components in the comoving frame of a mildly advective disk under the premise of negligible generation of meridional field (invoking Parker's undulate instability to promote the growth (loss) of vertical (horizontal) field is questionable in turbulent disks where the turbulence is fed by MRI's). A self-consistent turbulent steady state is achieved when the non-linear damping rate of the turbulent cascade equals the inverse of the linear growth timescale (Zhang, Diamond \& Vishniac 1994). Accretion disk models are far from being self-consistent in this respect.

The heuristic dynamo equations account for field generation by the shear flow and by the non-axisymmetric MRI in Paczyński's pseudo-potential well $\Phi \propto\left(r-r_{g}\right)^{-1}$ where $r_{g}=2 G M / c^{2}$; with flux buoyancy and turbulent reconnection providing for field loss terms. The model predicts azimuthally averaged field components and depends explicitly on the magnetic Mach number of the turbulence (assumed Alfvenic), on local pressure ratios, and on the relativistic generalization of the shear parameter $\left(\mathrm{A}_{\text {Oort }}^{\mathrm{Rel}}=\right.$ Oort's A constant $)$.

\section{DYNAMO EQUATIONS AND SCALINGS}

In Lagrangian coordinates

$$
\partial_{t} B_{\varphi}=\frac{B_{r}}{\tau_{\mathcal{S}}}-\frac{B_{\varphi}}{\tau_{\mathcal{B}}}-\frac{B_{\varphi}}{\tau_{r}} \quad \text { and } \quad \partial_{t} B_{r}=\frac{B_{\varphi}}{\tau_{\mathcal{M}}}-\frac{B_{r}}{\tau_{\mathcal{B}}}-\frac{B_{r}}{\tau_{r}} .
$$

The shear flow is parameterized linearly by a relativistic generalization of Oort's first constant (Novikov \& Thorne 1974), $\tau_{\mathcal{S}}^{-1}=2 \mathrm{~A}_{\text {Oort }}=\gamma^{2} d_{\ln r} \Omega$, where $\gamma$ is the bulk Lorentz factor of the flow. Shear forces the radial wavenumber of perturbations to evolve according to $k_{r}(t)=k_{r}^{0}-2 \mathrm{~A} k_{\varphi} t$.

We use exact analytical MRI scalings from Foglizzo \& Tagger (1995). With $\alpha_{\varphi} \equiv B_{\varphi}^{2} /(8 \pi p)$ and $\hat{\mathrm{A}} \equiv \mathrm{A} / \Omega<0$, the maximum growth of non-axisymmetric MRI modes occurs for wavenumber

$$
k_{\varphi} \equiv \eta \frac{\Omega}{v_{\varphi}^{\text {Alf }}} \quad \stackrel{\alpha_{\varphi} \ll 1}{\longrightarrow}\left\{2 \hat{\mathrm{A}}+\left(1+\alpha_{\varphi}\right) \hat{\mathrm{A}}^{2}\right\} \frac{\Omega}{v_{\varphi}^{\mathrm{Alf}}}
$$

at a rate, $\tau_{\mathcal{M}}^{-1}$, that obeys

$$
|\hat{\mathrm{A}}|^{2} \tau_{\mathcal{M}}^{2} \stackrel{\alpha_{\varphi} \ll 1}{\longrightarrow} 1+\alpha_{\varphi}(2+\hat{\mathrm{A}})
$$

as long as $k_{\varphi} \gtrsim 1 / r$. On the other hand, in a very strongly sheared flow

$$
-\hat{\mathrm{A}}>1+\left(2 \alpha_{\varphi}+1\right)^{-1},
$$

the slow branch of MHD propagation (to which the toroidal MRI belongs) is destabilized into a radial interchange mode (T. Foglizzo, Priv. Comm.) in accordance with the Rayleigh criterium. 
A plausible mechanism that promotes baryon unloading from field lines is turbulent pumping (Vishniac 1995a) by the MRI which must favorably compete with turbulent diffusion of matter back onto flux ropes. Under this assumption, the stretch, twist and fold of field lines by (enthalpy-weighted) sub-Alfvenic turbulence augments the field energy density and releases matter from field lines that otherwise would be "frozen-in". For the marginal case of Alfvenic turbulence, nearly empty $\mathrm{B}_{\perp}$ flux ropes (i.e. flux residing on surfaces perpendicular to the local meridian) in a gas pressure dominated disk acquire a drag limited buoyant velocity $v_{b} \propto\left(v_{\perp \theta}^{\text {Alf }}\right)^{2} / c_{s}$. Moreover, assuming efficient diffusion of radiation and $e^{ \pm}$pairs into the flux tubes, in this picture the buoyancy loss rate, $\tau_{\mathcal{B}}^{-1}=v_{b} / \mathcal{H}_{\theta}$, is enhanced by a factor $\lesssim p / p_{\text {gas }} \equiv \xi$ (Vishniac 1995b).

Reconnection of the field at sub-MRI optimal lengthscales (where the field lines are only weakly stochastic) occurs at the Alfvén speed for Alfvenic turbulence (Lazarian \& Vishniac 1999). The rates may be written as inverse Alfvén transit times calculated from the component of the field that undergoes reconnection,

$\tau_{\text {rec }} \approx l_{\text {rec }}^{\perp i} / v_{i}^{\text {Alf }}=\sqrt{2 / \Gamma}\left(l_{\text {rec }}^{\perp i} / \mathcal{H}_{\Theta}\right)\left(c_{s} / v_{i}^{\text {Alf }}\right) \Omega^{-1}$, where $\Gamma$ is the adiabatic index of the fluid.

The perpendicular lengthscales, $l_{\mathrm{rec}}^{\perp i}$, associated with the mean distance for field reversal are derived from the fundamental linear lengthscales for coherent field pumping. These are supplied by half of the toroidal MRI's (wave)length scale. Azimuthal, radial field reversal, $Y_{r}$, directly involves the optimal wavenumber of the non-axisymmetric MRI. In addition, following Tout \& Pringle (1992), an estimate of the radial, azimuthal field reversal lengthscale, $X_{\varphi}$ follows by noting that the time evolution of wavenumbers implied by shear during one MRI timescale couples the azimuthal lengthscale to the radial lengthscale, i.e. $l_{y}^{\perp}=\left(\tau_{\mathcal{M}} / \tau_{\mathcal{S}}\right) \times l_{x}^{\perp}$. Thus $Y_{r}=\pi / k_{\mathcal{M}}$ and $X_{\varphi}=\left(\tau_{\mathcal{S}} / \tau_{\mathcal{M}}\right) \times\left(\pi / k_{\mathcal{M}}\right)$.

\section{EQUILIBRIUM SOLUTIONS}

Scaling wavenumbers to the inverse pressure scale height $k \rightarrow \hat{k} / \mathcal{H}_{\Theta}$, and redefining $\hat{\mathrm{A}} \equiv|\mathrm{A}| / \Omega>0$; a set of normalized dynamo equations follows by representing fields in (Alfvén ) velocity units and normalized to the soundspeed $\left(B^{\prime}=B \times \sqrt{4 \pi \varrho} c_{s}\right)$, and the time normalized to the inverse of the Keplerian frequency $t^{\prime}=\Omega t$.

In a steady state, these equations must satisfy

$$
B_{r}^{\prime}=\frac{B_{\varphi}^{\prime}}{2 \hat{\mathrm{A}}}\left\{\frac{1}{\xi}{B_{\perp}^{\prime 2}}^{2}+\frac{\sqrt{2}}{\pi} \frac{\tau_{\mathcal{M}}^{\prime}}{\tau_{\mathcal{S}}^{\prime}} \eta\right\}, \text { and } B_{\varphi}^{\prime}=\frac{B_{r}^{\prime}}{\hat{\mathrm{A}}}\left\{\frac{1}{\xi}{B_{\perp}^{\prime 2}}^{2}+\frac{\sqrt{2}}{\pi} \frac{B_{r}^{\prime}}{B_{\varphi}^{\prime}} \eta\right\}
$$

which we solve numerically. 


\section{THE ENERGY DEPOSITION RATE}

The accretion disk setting is envisioned to follow the standard hyper-accreting black hole model of Popham, Woosley \& Fryer (1999, hereafter PWF) where $M_{\mathrm{bh}}=$ $3 \mathrm{M}_{\odot} ; \alpha_{\mathrm{SS}}=0.1$, and $\dot{M}=0.1 \mathrm{M}_{\odot} \mathrm{sec}^{-1}$. By adopting their published pressure ratios and assuming Keplerian rotation for $r \in[2.25,20] r_{g}$, we "piggyback" the hydromagnetic energy conversion process on this model.

We find that the magnetic output rate from buoyancy, $\dot{E} \simeq 1.77_{+51} \mathrm{erg} \mathrm{sec}^{-1}$, is comparable to the neutrino luminosity $L_{\nu} \simeq 3.3_{+51} \mathrm{erg} \mathrm{sec}^{-1}$ (PWF). The 'halfluminosity' radius is located at $r_{\mathcal{L}} \approx 5.75 r_{g}$ and the IDD becomes operational (against the radial interchange instability, c.f. Eq [4] and Araya-Gochez 1999) at

$r_{\min } \simeq 2.55 r_{g}$. Curiously, the derived value of the magnetic viscosity $\alpha_{\mathrm{SS}}=B_{r}^{\prime} B_{\varphi}^{\prime}$ hovers on 0.1 at the innermost radii and decreases to about .087 at $r_{\mathcal{L}}$ in good agreement with the adopted value. Lastly, we note that the MRI pumps the field preferably at the lowest wavenumbers for a near-equipartition field in a strongly sheared flow. Thus, most of this energy could in principle go into a Poynting jet with interesting consequences for $\gamma$-ray bursts.

\section{REFERENCES}

1. Araya-Gochez, R.A. 1999, M.N.R.A.S. (submitted) astro-ph/9912324

2. Davies et al. 1994, Ap.J., 431, 742

3. Foglizzo, T. \& Tagger M. 1995, A. \& A., 301, 293

4. Lazarian, A. \& Vishniac, E.T. 1999, Ap.J. 517, 700 (astro-ph/9811037)

5. MacFadyen \& Woosley 1998, Ap.J. 524, 262 (astro-ph/9810274)

6. Mészáros, P., \& Rees, M.J. 1997, Ap.J.Lett, 482, L29

7. Mészáros, P., Rees, M.J. \& Wijers, R.A. 1999, New Ast., 4, 303 (astro-ph/9808106)

8. Novikov, I. \& Thorne, K.S. 1973, in Black Holes, eds. C. DeWitt \& B.S. DeWitt (New York: Gordon \& Breach), 343

9. Popham, R., Woosley, S., \& Fryer, C. 1999, Ap.J., 518, 356 (astro-ph/9807028)

10. Rasio, F.A. \& Shapiro S.L. 1994, Ap.J., 432, 242.

11. Ruffert \& Janka 1999, A\&A 344, 573 (astro-ph/9809280)

12. Thompson, C. 1994, M.N.R.A.S., 270, 480

13. Tout, C.A. \& Pringle, J.E. 1992, M.N.R.A.S., 259, 604; 1995, M.N.R.A.S., 281, 219

14. Vishniac, E.T. 1995a, Ap.J., 446, 724; 1995b, Ap.J., 451, 816

15. Zhang, W., Diamond P.H. \& Vishniac, E.T. 1994, Ap.J., 420, 705 